\title{
Pinning method of pulse confinement in optical fiber with random dispersion
}

\author{
M. Chertkov, I. Gabitov, P. M. Lushnikov, J. Moeser, and Z. Toroczkai \\ Theoretical Division, Los Alamos National Laboratory, Los Alamos, New Mexico 87545
}

Received August 17, 2001; revised manuscript received May 6, 2002

\begin{abstract}
Pulse propagation in optical fiber links with randomness in the dispersion is investigated theoretically and numerically for systems with and without dispersion management. The main effects of locally correlated noise are inevitable pulse destruction and statistical broadening. However, periodic/quasi-periodic management of fiber randomness, achieved by setting the accumulated dispersion to its nominal value (pinning), essentially decreases pulse broadening. If the pinning period is short enough, we observe a statistical state that is numerically indistinguishable from the steady state in the dispersion-managed case. (c) 2002 Optical Society of America

OCIS codes: $060.2330,060.4370,060.4510,060.5530$.
\end{abstract}

\section{INTRODUCTION}

A decade ago, in the early stages of high-speed optical communication, the debate over linear or nonlinear (soliton) supported optical transmission was heated. At the time, the existing fiber communication infrastructure was based on linear transmission. The theoretical ${ }^{1}$ and experimental (see, for example, Refs. 2-4) results for nonlinear transmission showed that the performance of these systems could be better than that for linear systems. However, the performance improvement was not dramatic enough to merit a complete overhaul of the existing linear systems. With the invention ${ }^{5}$ and recent testing $^{6-9}$ of a new type of nonlinear supported transmission, dispersion management, there remains little doubt about the effectiveness of communications based on nonlinear principles.

With today's increasing demand for higher bandwidth, the next challenge in optical communication is dealing with randomness of optical systems. It is crucial to take into account fiber system randomness when describing the evolution of the short pulses needed for higher-bitrate communication. The straightforward approach of refining production technology is expensive and does not offer an absolute cure. As a result, this subject has been the focus of many recent experimental and theoretical investigations, ${ }^{10-12}$ with the eventual goal being improved system performance in the presence of randomness. This problem is nontrivial even for linear systems and is not well understood in nonlinear systems.

Disorder in single-mode fibers arises in many different ways and has a negative effect. For example, amplifier noise, ${ }^{13-16}$ and random fiber birefringence (polarization mode dispersion $)^{17-20}$ lead to random shifts in the pulse position (timing jitter) and pulse broadening. Both effects eventually cause destruction of bit patterns and lead to an increase of the bit error rate, the most important parameter describing performance in fiber communications systems. $^{21}$ The description of data-stream degradation requires the use of statistical methods and opens a new field that may be called statistical physics of fiber-optic communication.

In the present paper, we consider the effects caused by the randomness of the fiber chromatic dispersion. Recent high-precision measurements of this dispersion demonstrated the significance of the dispersion randomness. ${ }^{22,23}$ Chromatic dispersion is an important characteristic of a medium and can significantly degrade the integrity of wave packets. In practice, chromatic dispersion is not uniformly distributed and often exhibits random variations in space. This disorder can be treated as multiplicative noise in corresponding mathematical models. This multiplicative noise is conservative, as the wave energy remains constant during propagation through the medium. The overall chromatic dispersion in optical fibers comes from two sources. The first source is the medium itself. The second source is due to specific geometry of the waveguide profile. Material dispersion in the optical fiber is a relatively stable parameter, uniformly distributed along the fiber. However, the waveguide dispersion is not nearly as stable. Existing technology does not provide accurate control of the waveguide geometry in modern fibers, in which dependence of the dispersion coefficient on the wavelength is complex. As a result, the magnitudes of random variations of fiber chromatic dispersion are typically the same as, or in some cases even greater than, that of the mean dispersion ${ }^{22,23}$ in fibers operating near the zero-dispersion point (dispersion-shifted fibers).

The natural problems stemming from randomness in chromatic dispersion are pulse degradation and bitpattern deterioration. We focus on the pulse degradation due to propagation through an optical fiber with a Gaussian white-noise component in its chromatic dispersion. The problem of degradation might be overcome by perfecting the processes of fiber pulling from a silica preform and cabling, and by compensating for the effects of randomness. This compensation can be active or passive. Active compensation assumes individual preshaping of a pulse or sequence of pulses to minimize the pulse change 
during transmission through a specific fiber link. ${ }^{24}$ This technique has great potential for ultrashort-pulse delivery over short distances. Nonuniversality of the active approach is its major drawback. The idea of a passive approach is to modify the line itself by inserting additional system components. Passive compensation offers a greater degree of flexibility, as it works for a variety of signal formats and pulse shapes.

In this paper, we propose a passive strategy, the pinning method, which prevents pulse degradation. Pinning is the periodic/quasi-periodic compensation of the random part of the accumulated fiber dispersion. Pinning is capable of significantly reducing the pulse deterioration (reducing bit error rate) caused by the disorder. Furthermore, the method may even provide statistically steady propagation of the pulse along the fiber.

The material in this paper, which is an extended version of the short letter, ${ }^{25}$ is organized as follows. The problem is formulated in Section 2. There we introduce the nonlinear Schrödinger model with fluctuating dispersion. The dispersion coefficient consists of deterministic and random parts. We consider two models for the deterministic part: (A) a constant positive dispersion; (B) a piecewise constant dispersion with positive residual value (dispersion management). Both natural and pinned randomness are considered. In Subsection 2.A we give the statistical framework for description of the single-pulse evolution. Subsection 2.B defines a synthetic modification of the natural disorder (passive compensation of accumulated random dispersion by the pinning method). Section 3 discusses the weak nonlinearity limit. The path-averaged approach describing slow-pulse dynamics is introduced. It is shown that the natural disorder leads to inevitable destruction of the pulse integrity. In this case the signal envelope is a strongly fluctuating object, which prevents the introduction of a deterministic equation describing the dynamics of the signal envelope. In the case of pinning, however, the dynamics of the signal envelope are shown to be described by a deterministic path-averaged equation. Subsection 3.A is devoted to the numerical demonstration that the averaged equation does have a steady solitonlike solution for both models $\mathrm{A}$ and B. The probability distribution functions (PDFs) of the pulse width and amplitude described in Subsection 2.A are studied numerically in Section 4. A decrease in the rate of the pulse degradation in the pinned case is observed. The dependence of the degradation on the pinning length and on the disorder strength is investigated. The final section contains our conclusions.

\section{FORMULATION OF THE PROBLEM}

In the short-wavelength regime, a universal description of the signal envelope in the reference frame moving with the packet group velocity is given by the nonlinear Schrödinger equation (NLS) for the complex scalar field, $\psi(z ; t)$ (see, for example, Ref. 21),

$$
-i \partial_{z} \psi=\mathrm{d}(z) \partial_{t}^{2} \psi+2 \psi^{2} \bar{\psi} .
$$

All parameters and coefficients of Eq. (1) are presented in dimensionless units that transform to real-world fiber units according to the following rules. The envelope $\psi$ of the electric field is in the form $E=\operatorname{Re}\left[\psi \sqrt{P_{0}} \exp (i c t / \lambda)\right]$, where $P_{0}$ is the peak pulse power, $\lambda$ is operating wavelength, $c$ is velocity of light, and $E$ is measured electric field. The propagation variable is $z=x\left(\alpha P_{0} / 2\right)$, where $x$ is distance along the fiber and $\alpha$ is the Kerr nonlinearity coefficient. The Kerr coefficient can be expressed in terms of other fiber parameters, $\alpha=2 \pi n_{2} /\left(\lambda S_{\text {eff }}\right)$, where $n_{2}$ is the nonlinear component of fiber refractive index and $S_{\text {eff }}$ is an effective core area of the fiber. The spatial coordinate is $t=\tau / \tau_{0}$, where $\tau$ is in the reference frame of the group velocity and $\tau_{0}$ is the characteristic pulse width. The dispersion coefficient is $d=2 \beta_{2} /\left(\alpha P_{0} \tau_{0}^{2}\right)$, where $\beta_{2}$ is the second-order dispersion parameter. Typical parameters for the dispersion-shifted fiber are $\lambda=1550 \mathrm{~nm}, \quad \tau_{0}=7 \mathrm{ps}, x=50 \mathrm{~km}, P_{0}=4 \mathrm{~mW}, \beta_{2}$ $=2 \mathrm{ps}^{2} / \mathrm{km}$, and $\alpha=10 \mathrm{~W}^{-1} \mathrm{~km}^{-1}$.

Variations in the medium (fiber) enter Eq. (1) through the dispersion coefficient

$$
d(z)=d_{\operatorname{det}}(z)+\xi(z)
$$

which is decomposed into deterministic, $d_{\text {det }}(z)$, and random (disorder), $\xi(z)$, parts. Here $z$ is the position along the fiber and $t$ is the retarded time. The initial profile $\psi(0 ; t)$ is localized in $t$.

We consider two different models of deterministic dispersion. Both models are standard in fiber-optics communications.

Model A is the case of constant dispersion, $d_{\mathrm{det}}=d_{0}$. In the absence of noise $[\xi(z)=0], \quad \psi_{0}(z ; t)$ $=a \exp \left(i z d_{0} / b^{2}\right) \operatorname{sech}(t / b)$, where $a^{2} b^{2}=d_{0}(a$ is the peak amplitude, and $b$ is the pulse width), is an exact singlesoliton solution of Eq. (1). The existence of the soliton ${ }^{26,27}$ is the result of a dynamic equilibrium between dispersion and nonlinearity: the two spatial scales, of nonlinearity $z_{\mathrm{NL}}=1 / a^{2}$ and of dispersion $z_{d}=b^{2} / d_{0}$, coincide.

Model B is the case of dispersion management (DM), $d_{\text {det }}=d_{0} \pm d_{\mathrm{DM}} \cdot{ }^{5}$ Here dispersion is piecewise constant: positive and negative spans alternate with period $z_{\mathrm{DM}}$. There is no exact solution known for the pure (no noise) model B, but theoretical evidence, confirmed by extensive numerical studies and experimental results, indicates the existence of a breathing solution (DM soliton) with a nearly Gaussian shape. ${ }^{28-31}$ The localized solution here is again due to the interplay of dispersion and nonlinearity. In the presence of a periodic dispersion map, however, the (DM) soliton acquires an important characteristic, quadratic phase (chirp). In contrast to conventional soliton solutions, DM solitons can exist for zero (or even negative) values of the average dispersion. ${ }^{32,33}$

Approximate scale characteristics of the dispersion noise present in real fibers can be extracted from experimental results. ${ }^{22,23}$ These results show that the typical distance of noticeable change in the dispersion value, $z_{\mathrm{var}}$, is shorter than $\sim 1-2 \mathrm{~km}$. (The resolution of the experimental method is $1-2 \mathrm{~km}$, whereas one expects that the typical scale of the variations is actually one to two orders of magnitude shorter, $\sim 10-100 \mathrm{~m}$, which is the size of the production facility.) For constant-dispersion fibers (model A), the amplifier spacing is $\sim 50-60 \mathrm{~km}$, and for dispersion-managed fibers (model B), the period of a typical dispersion map is also $\sim 50-60 \mathrm{~km}$. These scales 
are much longer than that of the dispersion variation. Therefore, according to the central limit theorem, ${ }^{34}$ the natural $\xi$ at the larger scales can be treated as a homogeneous Gaussian random process with zero mean, described by the quantity, $D=\int \mathrm{d} z\left\langle\xi(z) \xi\left(z^{\prime}\right)\right\rangle$. The noise intensity, $D$, is defined as $z_{\text {var }} d_{\text {var, }}^{2}$, where $d_{\text {var }}$ is the typical amplitude of the fluctuating part of the dispersion variations. Therefore the pair correlation function of $\xi$ is given by

$$
\left\langle\xi\left(z_{1}\right) \xi\left(z_{2}\right)\right\rangle=D \delta\left(z_{1}-z_{2}\right)
$$

(Here and below, $\langle Q\rangle$ stands for the disorder average of the quantity $Q$.) Previously, the stability of a pulse in the presence of the natural noise was studied for both models (A and B) numerically and by means of a variational approach. $^{35-37}$ The unambiguous conclusion of these studies was that a localized pulse does not survive propagation, i.e., it is destroyed. The numerical study of the natural noise, Eq. (3), explained below in Section 4, confirms the early observations. In Appendix A we also provide a rigorous proof that steady propagation is not possible in the case of model $\mathrm{A}$ and nonpositive residual dispersion $d_{0}$ (the average pulse width grows monotonically with distance). We also discuss in Appendix A rigorous constraints on averages, characterizing dynamical evolution of an initially localized pulse in various regimes studied.

\section{A. Statistical Approach: Probability Distribution Functions and Averages}

We present the statistical framework necessary for describing single-pulse propagation through a noisy medium. If the pulse is distinguishable from the radiative background, its temporal profile can be characterized by a finite number of degrees of freedom. This number may change with propagation in $z$, as is the case when one of the solitons vanishes. The pulse separation from the background radiation is obvious in the weak-disorder case of model A. Indeed, the soliton solution is known exactly in the no-noise limit of model A. In the presence of weak noise, the pulse evolution can be described in the framework of the adiabatic approximation. This approximation is characterized by the slow evolution of four modes: width, phase, position, and phase velocity of the pulse. At least two of the modes, position and width, are general, in that they can be used to describe pulse evolution in a regime far from that of weak noise. In the strong-noise regime, it is also sensible to characterize the pulse by its amplitude, which evolves independently of the width. This contrasts with the adiabatic case, in which width and amplitude are strongly related. Since we consider only symmetric single pulses, the pulse position is not a relevant parameter of interest. Therefore in this paper we consider only two parameters, the width and the amplitude of the pulse.

In this context, the key objects of statistical analysis are the probability distribution functions (PDFs) of the pulse width, $b$, and amplitude, $a$, where statistics are collected over the ensemble of the dispersion map realizations described by Eqs. (3) (natural disorder) and (4) (disorder synthetically constrained by pinning). In general, one expects that these PDFs will evolve with $z$. Analysis of the linear case, including evolution of the PDFs, is given in Appendix B.

The adiabatic (weak noise) limit $(D \ll 1)$ for model A was described recently in Ref. 38. Here the solution description by the adiabatic approximation is actually deterministic: in the leading order the PDFs are $\delta$ functions, with the width of the pulse being the inverse of its amplitude. The dependence of the (averaged) amplitude on $z$ (at $\left.d_{0}=1\right)$ is given by $a(z)=(1+8 D z / 3)^{-1 / 5}$. The effect of noise is realized through the shedding of radiation by the pulse.

In the general case ( $D$ not necessarily small) for natural (unpinned) noise, the average value of the pulse amplitude decreases. Furthermore, the PDFs of the pulse parameters widen and are no longer narrow functions of their arguments. These statistics of degradation and broadening of the pulse are studied numerically in Section 4.

However, in the pinned version of model B (see the next subsection for the definition of pinning) with the pinning length being shorter than the correlation scale of the pulse degradation (defined in Section 3), the PDFs of the pulse amplitude and width approach a stationary limit. This is described in Section 4, where a numerical study of the PDFs is presented.

\section{B. Pinning Method}

In the case of strong noise, a natural step would be to develop an artificial constraint capable of reducing or completely preventing pulse destruction and broadening. We demonstrate that such a constraint does indeed exist and can be readily implemented in real fibers. The necessary constraint is that the accumulated dispersion, $\int_{0}^{z} \mathrm{~d} y \xi(y)$, is set to zero, or pinned, either periodically or quasiperiodically with a period of the order of (or less than) that of the pulse degradation length, $z_{\xi}$.

We describe the theoretical basis for the pinning method in this subsection (the degradation length of a pulse in the natural-disorder case, $z_{\xi}$, is defined in Section 3). In the case of pinning, $\xi$ is replaced by $\xi_{p}$, which is no longer a delta function, and is instead characterized by the pair-correlation function

$$
\left\langle\xi_{p}(y) \xi_{p}(z)\right\rangle=D\left[\delta(z-y)-\frac{1}{l_{j+1}-l_{j}}\right],
$$

where $y$ and $z$ belong to the same segment bounded by an adjacent pair of pinning points, i.e., $l_{j}<y, z<l_{j+1}$, where $j$ is the pinning-point label. Otherwise, correlations vanish, i.e., $\left\langle\xi_{p}(y) \xi_{p}(z)\right\rangle=0$. The pinned process, Eq. (4), can be constructed from the natural one, Eq. (3), by

$$
\xi_{p}(z)=\xi(z)-\frac{1}{l_{i+1}-l_{i}} \int_{l_{i}}^{l_{i+1}} \mathrm{~d} y \xi(y),
$$

This nonuniform noise satisfies the pinning restriction $\int_{l_{i}}^{l_{i+1}} \xi_{p}(y) \mathrm{d} y=0$ on each and every dynamical realization.

The pinning of noise according to Eq. (4) suggests a new strategy for the production of new optical fiber cables: controlling the integral dispersion of a fiber piece 
before its connection to other pieces. The control is achieved, first, by accurate measurement of the fiber's dispersion profile (the method of Ref. 22 is ideal for the purpose), second, by the identification of zeros for $\int_{0}^{z} \mathrm{~d} z^{\prime} \xi\left(z^{\prime}\right)$, and finally, by the cutting of the fiber at one of the zeros.

There also exists another pinning strategy, point pinning, which is the local insertion of short compensating pieces. It is described by

$$
\xi_{p p}(z)=\xi(z)-\sum_{j} \delta\left(z-l_{j}\right) \int_{l_{j-1}}^{l_{j}} \mathrm{~d} z^{\prime} \xi\left(z^{\prime}\right)
$$

Point pinning can be used for the improvement of system performance in already installed fiber lines. All the results reported in this paper were obtained with the first pinning strategy. Nevertheless, they apply equally to point pinning. Moreover, from the point of view of the adiabatic analysis of Ref. 38, for $z \gg 1$ both the models with the equidistantly positioned pinning points $\left(l_{i+1}\right.$ $-l_{i}=l$ for any $i$ ) have the same "continuous" limit $(l \rightarrow 0)$, described by $\left\langle\xi_{p}(z) \xi_{p}\left(z^{\prime}\right)\right\rangle=-D l^{2} \delta^{\prime \prime}(z$ $\left.-z^{\prime}\right) / 12$.

The pinning method has some predecessors, both theoretical and experimental. Ohhira and coauthors ${ }^{39}$ have considered propagation of a pulse through a fiber with piecewise constant dispersion. The dispersion of a single span (each of the same length) was taken to be a random Gaussian number with nonzero mean. It was shown, mainly by numerical means, that the propagation improves if the spans are ordered in an alternating descendant manner. (The span with the largest positive value should be followed by the one with the largest negative one, with the next pair chosen according to the same principle from the bank of remaining spans.) The approach of Ref. 39 did not account for the short correlated disorder (reported later in Ref. 22). The functional disorder ( $\xi$ is a random function of $z$ ) in dispersion (of a general kind considered in our paper) is reduced in Ref. 39 to fluctuations of a single parameter (accumulated dispersion of a fiber span). The improvement of propagation achieved in Ref. 39 by reordering the sequence of fiber spans is therefore a very special way to enforce the pinning of accumulated dispersion.

In an experimental simulation of long-haul data transmission, Mollenauer and coauthors ${ }^{9}$ put a number of fiber spans together, each span consisting of a combination of different fibers in order to design a specific dispersion profile. The whole system was looped to simulate transmission over sizable distances. The measurement of the values of accumulated dispersion of each span, which were presumed to be the same initially, showed the presence of irregularity. It was shown that the insertion of an extra piece of fiber into each span, compensating for the irregularity, improves the transmission. Randomness (or averaging) was not an issue in Ref. 9: a deterministic process (i.e., one complex fiber) was studied. Therefore the observation of Ref. 9 is an ancestor ("deterministic" one) of the accumulated dispersion pinning discussed in our paper.

\section{WEAK NONLINEARITY: PATH- AVERAGED INTEGRAL EQUATION}

The large dynamical variation of certain system parameters about a mean value creates the temptation to perform an averaging procedure with respect to these oscillations. In the context of the NLS, with a dispersion coefficient that varies periodically with $z$, such a method is known under the name of path averaging. ${ }^{28}$ In this section we investigate the possibility of generalizing this method to the case of random variations in dispersion.

The assumption of quasi linearity is the core of the path-averaging approach. This assumption suggests the following substitution:

$$
\begin{aligned}
\psi(z ; t)= & \int_{-\infty}^{\infty} \mathrm{d} \omega \exp [-i(\omega t \\
& \left.\left.+\omega^{2}\left\{\int_{0}^{z}\left[\xi\left(z^{\prime}\right)+d_{\operatorname{det}}\left(z^{\prime}\right)-d_{0}\right] \mathrm{d} z^{\prime}\right\}\right)\right] \phi_{\omega}(z) .
\end{aligned}
$$

If the nonlinearity is neglected, $\phi_{\omega}$ experiences only dispersive broadening governed by $d_{0}$. One assumes that the nonlinearity is weak. (The fluctuations in dispersion are strong.) Then the fast part of the $\psi$ dynamics is already accounted for in the oscillating kernel of Eq. (7), and the slow spatial dynamics (in $z$ ), due to $d_{0}$ and weak nonlinearity, are described by the evolution of $\phi_{\omega}$. Substitution of Eq. (7) into Eq. (1) results in

$$
\begin{aligned}
-i \partial_{z} \phi_{\omega}+ & d_{0} \omega^{2} \phi_{\omega} \\
= & 2 \int_{-\infty}^{\infty} \delta\left(\omega_{1}+\omega_{2}-\omega_{3}-\omega\right) \mathrm{d} \omega_{1} \mathrm{~d} \omega_{2} \mathrm{~d} \omega_{3} \phi_{1} \phi_{2} \bar{\phi}_{3} \\
& \times \exp \left\{-i \Delta \int_{0}^{z}\left[\xi\left(z^{\prime}\right)+d_{\operatorname{det}}\left(z^{\prime}\right)-d_{0}\right] \mathrm{d} z^{\prime}\right\},
\end{aligned}
$$

where

$$
\Delta=\omega_{1}^{2}+\omega_{2}^{2}-\omega_{3}^{2}-\omega^{2}, \quad \phi_{i} \equiv \phi_{\omega_{i}}, \quad i=1,2,3 .
$$

A common, but not always justified, approach is to assume that $\phi_{\omega}$ is a self-averaging quantity at large $z[z$ should be essentially larger than the typical scale of variations in $d(z)$ ]. A way to check this hypothesis is to study the integral of the kernel in Eq. (8):

$$
\begin{aligned}
& \mathcal{F}(\Delta ; z) \\
& \quad \equiv z^{-1} \int_{0}^{z} \mathrm{~d} z_{1} \exp \left\{-i \Delta \int_{0}^{z_{1}}\left[\xi\left(z^{\prime}\right)+d_{\operatorname{det}}\left(z^{\prime}\right)-d_{0}\right] \mathrm{d} z^{\prime}\right\} .
\end{aligned}
$$

If the fluctuations of $\mathcal{F}$ due to randomness are suppressed as $z \rightarrow \infty$, and $\mathcal{F}$ becomes an essentially deterministic object, then $\phi$ is a self-averaged object, which at large $z$ can be replaced by its average, $\varphi \equiv\langle\phi\rangle$. In this case the averaged field, $\varphi \equiv \bar{\phi}$, satisfies the deterministic equation

$$
\begin{aligned}
-i \partial_{z} \varphi_{\omega}+d_{0} \omega^{2} \varphi_{\omega}= & 2 \int_{-\infty}^{\infty} \delta\left(\omega_{1}+\omega_{2}-\omega_{3}-\omega\right) \\
& \times \mathrm{d} \omega_{1} \mathrm{~d} \omega_{2} \mathrm{~d} \omega_{3} \mathcal{F}(\Delta ; z) \varphi_{1} \varphi_{2} \bar{\varphi}_{3} .
\end{aligned}
$$


If the noise is unpinned, $\mathcal{F}$ does not pass the selfaveraging test. Indeed, in the case of model A one obtains

$$
\begin{aligned}
\langle\mathcal{F}(\Delta ; z)\rangle= & \frac{2}{\Delta^{2} D z}\left[1-\exp \left(-\frac{\Delta^{2} D z_{1}}{2}\right)\right], \\
\left\langle\mathcal{F}(\Delta ; z)^{2}\right\rangle= & \frac{1}{\Delta^{4} D^{2} z^{2}}\left[1-\frac{4}{3} \exp \left(-\frac{\Delta^{2} D z}{2}\right)\right. \\
& \left.+\frac{1}{3} \exp \left(-2 \Delta^{2} D z\right)\right]
\end{aligned}
$$

and at $z \rightarrow \infty,\left\langle\mathcal{F}^{2}\right\rangle \neq\langle\mathcal{F}\rangle^{2}$. The argument for model B is similar. Therefore one concludes that the deterministic path-averaged approach is not applicable to naturalnoise cases. However, a useful lesson can still be drawn from this grim conclusion: the nonlinear kernel decays with $z$ (because all of its positive integer moments decay). Thus the residual dispersion dominates, and as a result the pulse broadens and degrades. The physical reason for the degradation is the loss of correlations: even though the random noise returns to zero regularly with increasing $z$ (the number of zero crossings for the noise grows linearly with $z$ ), its integral, which is actually the object in the kernel, does not. As shown in Appendix C, the number of returns to zero for the integral of the (natural) random process grows as $\sim \sqrt{z}$, slower than linear. Thus for increasing $z$ there are fewer chances (per unit length) of observing a value of $\int_{0}^{z} \mathrm{~d} z^{\prime} \xi\left(z^{\prime}\right)$ close to zero. Therefore Eqs. (12) and (13) also provide useful information: the length of the pulse destruction, which is the scale of the kernel decay in Eq. (11), is

$$
z_{\xi}=b^{4} / D
$$

where $b$ is the pulse width.

Note that effect of pulse deterioration in fiber links due to random variations of fiber chromatic dispersion increases rapidly at higher bit rates $\left(\mathrm{BR} \sim b^{-1}\right), z_{\xi}$ $\sim\left(D \times \mathrm{BR}^{4}\right)^{-1}$, and might therefore cause serious limitations for high-speed fiber communications. For the case of the fiber sample measured in the paper, ${ }^{22,9}$ such deterioration becomes noticeable at the distance $\sim 100 \mathrm{~km}$ for the pulse width $\tau_{0} \sim 2 \mathrm{ps}$.

A way to reduce the decay of the kernel is to implement one of the previously mentioned pinning strategies. If we force $\int \xi$ to return to zero periodically or quasi periodically, $\mathcal{F}$ is self-averaged as $z \rightarrow \infty$, and in the case of the strictly periodic pinning with period $l$ it approaches

$$
\begin{aligned}
\mathcal{F}(\Delta ; z) \rightarrow & \left\langle\operatorname { e x p } \left\{-\frac{\Delta^{2} D z(l-z)}{2}\right.\right. \\
& \left.\left.+i \int_{0}^{z} \mathrm{~d} z^{\prime}\left[d_{\operatorname{det}}\left(z^{\prime}\right)-d_{0}\right]\right\}\right\rangle_{z \gg l},
\end{aligned}
$$

$($ for model A $)=\exp \left(-\frac{\Delta^{2} D l}{4}\right) \sqrt{\frac{\pi}{\Delta^{2} D l}} \operatorname{Erfi}\left(\frac{\sqrt{\Delta^{2} D l}}{4}\right)$.
Substituting the result of Eq. (16) into Eq. (11), one arrives at the desired generalization of the path-averaged equation valid only if pinning is applied.

The next subsection is devoted to the analysis of the path-averaged equation, justified if pinning is applied and also if the following asymptotic hierarchy of scales (spelling the weakness of nonlinearity) is obeyed, $z_{\mathrm{NL}} \sim 1 / a^{2}$ $\gg z_{\xi}, l$.

A. Numerical Solution of the Path-Averaged Equation In this subsection we show numerically that the pathaveraged equation (11) has a stationary solution of a soliton kind. To solve Eq. (11), we use a generalization of the numerical method for solving nonlinear integral equations that was recently suggested by one of the authors ${ }^{40}$ (initially for the pure DM problem). The main obstacle in a numerical evaluation of the path-averaged equation (11) is the nonlocality of the nonlinear term [right-hand side of Eq. (11)]. We denote here this term by $R(\omega)$. Numerical computation of $R(\omega)$ generally requires $N^{3}$ operations per iteration, where $N$ is a number of grid points in $\omega$ or $t$ space. There exists, however, a much more efficient numerical algorithm for calculation of $R(\omega)$. The only assumption is the existence of a Fourier transform: $\mathcal{F}(\Delta ; z)=1 / 2 \pi \int \mathrm{d} s \hat{\mathcal{F}}(s) \exp (i s \Delta)$, where $s$ is an auxiliary variable. One derives

$$
\begin{aligned}
R(\omega)= & \frac{1}{\pi} \int \mathrm{d} s \hat{\mathcal{F}}(s) \exp \left(-i s \omega^{2}\right) \int \delta\left(\omega_{1}+\omega_{2}-\omega_{3}\right. \\
& -\omega) \mathrm{d} \omega_{1} \mathrm{~d} \omega_{2} \mathrm{~d} \omega_{3} \varphi_{1}^{(s)} \varphi_{2}^{(s)} \bar{\varphi}_{3}^{(s)}
\end{aligned}
$$

where $\varphi^{(s)}(\omega) \equiv \varphi(\omega) \exp \left(i s \omega^{2}\right)$. In the $t$-space representation this expression takes the form

$$
\hat{F}^{-1}(R(\omega))=2 \int \mathrm{d} s \hat{\mathbf{G}}^{(s)}\left(\Psi^{(s)}(t)\right),
$$

where $\Psi^{(s)}(t) \equiv\left|\varphi^{(s)}(t)\right|^{2} \varphi^{(s)}(t)$ and $\hat{\mathbf{G}}^{(s)}$ is an integral operator corresponding to the multiplication operator $\hat{\mathbf{G}}^{(s)}\left(\left(\hat{\Psi}^{(s)}(\omega)\right) \equiv \exp \left(-i s \omega^{2}\right) \hat{\Psi}^{(s)}(\omega)\right.$ in $\omega$ space. Equations (17) and (18) suggest the following four-step numerical procedure to calculate $R(\omega)$ :

(i) the backward Fourier transform of $\varphi^{(s)}(\omega)$ $=\varphi(\omega) \exp \left(i s \omega^{2}\right)$ for every value of $s$;

(ii) a calculation of $\Psi^{(s)}(t)$ from $\varphi^{(s)}(t)$;

(iii) the forward Fourier transform of $\Psi^{(s)}(t)$;

(iv) a numerical integration (summation) of $\exp \left(-i s \omega^{2}\right) \hat{\Psi}^{(s)}(\omega)$ over $s$ for every value of $\omega$.

If fast Fourier transforms are used for steps (i) and (iii), the number of operations required increases as $N \log _{2} N$. The total number of operations for one iteration is $\sim 2 M N \log _{2}(N)$, where $M$ is the number of grid points for integration over $s$. We used the following typical values for our numerical solution of Eq. (11): $N=4096 ; M$ $=800$. One iteration of the (i)-(iv) scheme on an Alpha $500-\mathrm{MHz}$ workstation requires $\sim 10 \mathrm{~s}$ for double precision versus $40 \mathrm{~h}\left(4096^{3}\right.$ operations $)$ for the brute-force scheme.

The results of calculations are presented in two figures, one for model A and one for model B. (The parameters are $d_{0}=1$ in model $\mathrm{A}$, and $d_{0}=0.15, d_{\mathrm{DM}}=0.1$, and $z_{\mathrm{DM}}=1$ in model B.) Only the case of periodic pinning is 

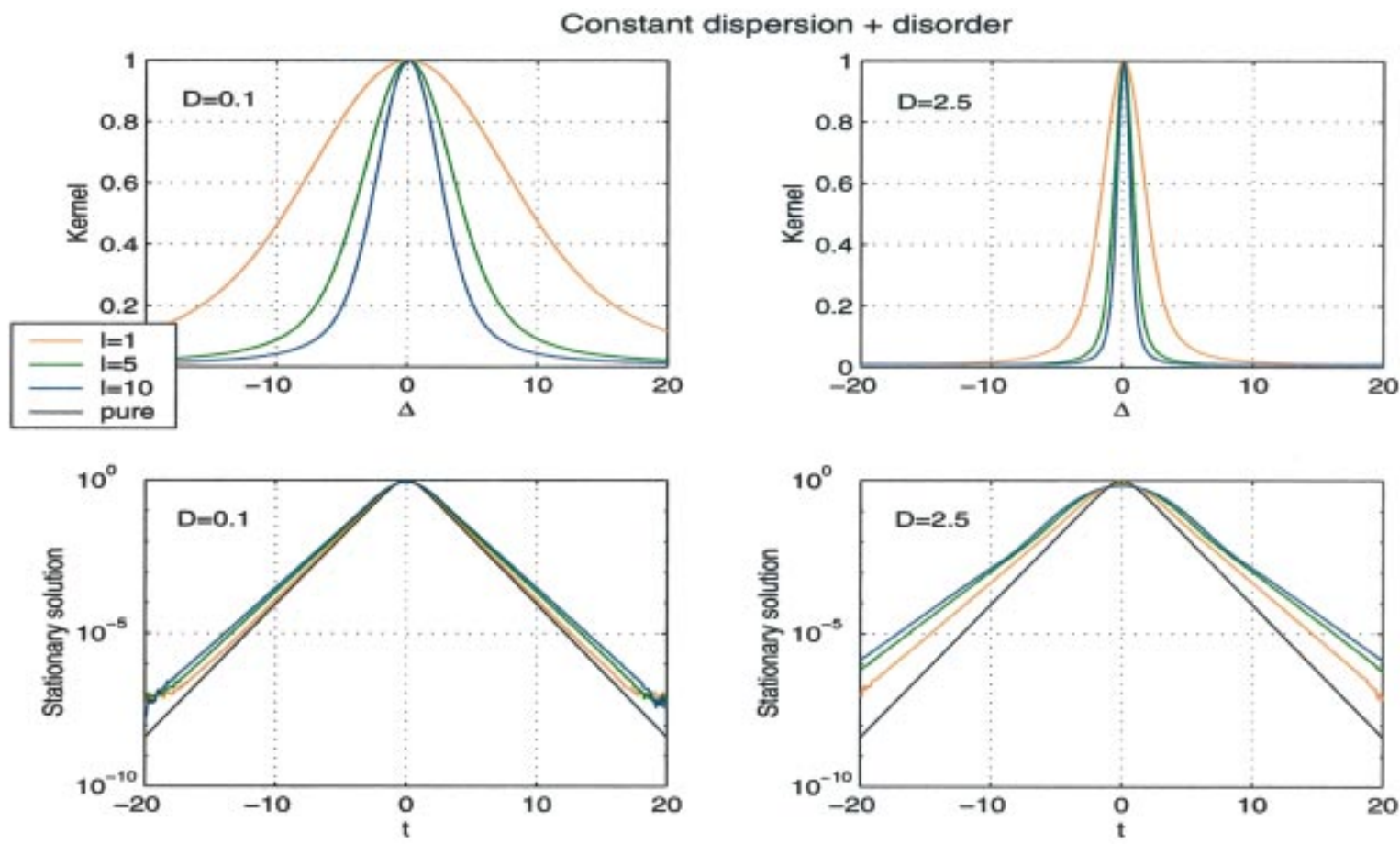

Fig. 1. Kernels and stationary solution shapes for the path-averaged equation: constant-dispersion case (model A). The upper row shows the shape of the kernel (16) for various pinning lengths. The lower row shows the corresponding stationary solutions (log scale) for the path-averaged equation. The pictures in the left column are for noise strength $D=0.1$, and those on the right are for $D=2.5$
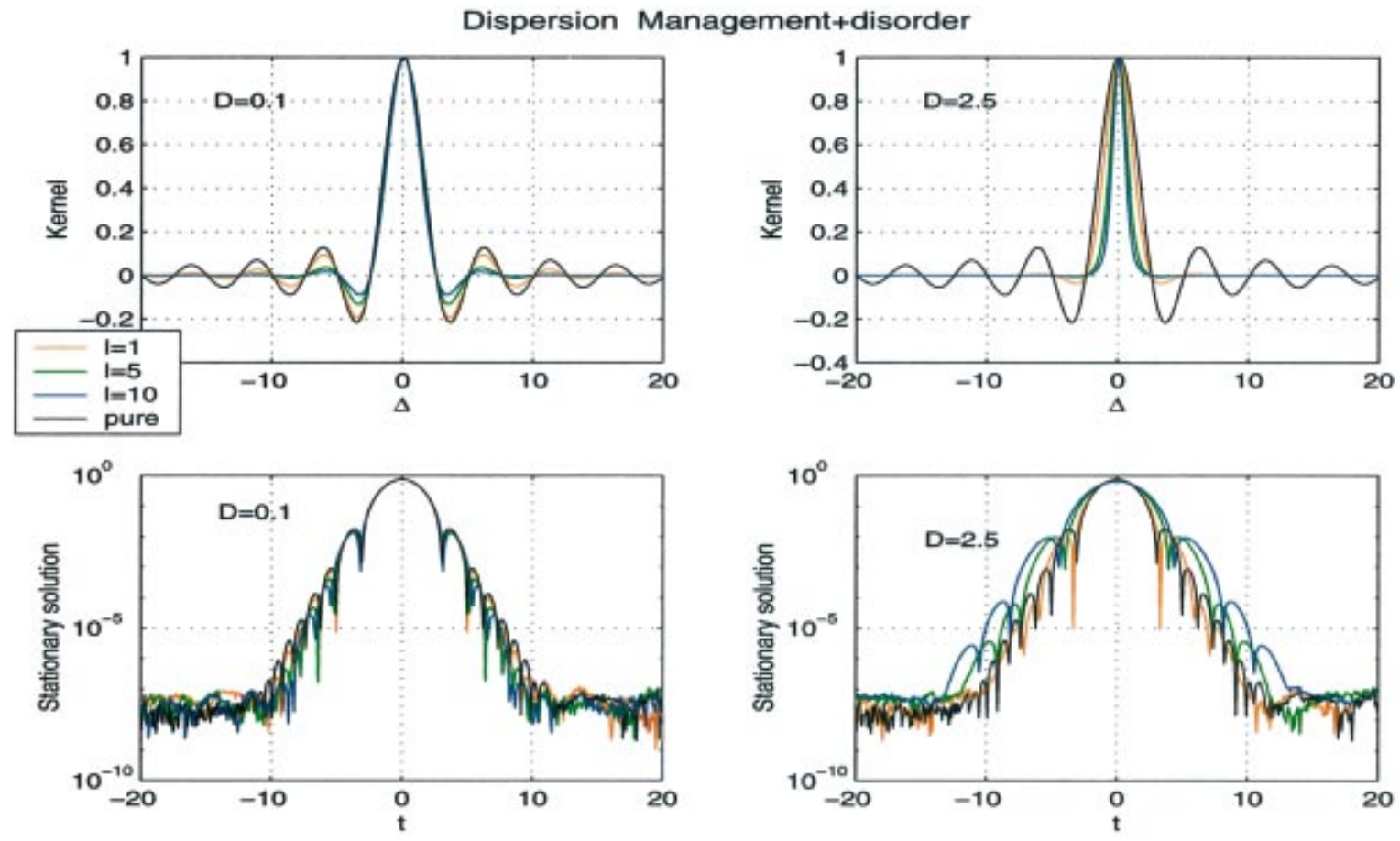

Fig. 2. Kernels and stationary solution shapes for the path-averaged equation: dispersion-management case (model B). The upper row shows the shape of the kernel for various pinning lengths. The lower row shows the corresponding stationary solutions (log scale) for the path-averaged equation. The pictures in the left column are for noise strength $D=0.1$, and those on the right are for $D=2.5$.

considered. Different colors correspond to different pinning lengths. The black curves stand for the pure case of a NLS soliton and a DM soliton, respectively (no noise).
Simulations in both cases were carried out for two values of the noise strength, $D=0.1$ and $D=2.5$. The upper plots in each set of figures (case A and case B) show pro- 
files of the kernel of the path-averaged equation as a function of $\Delta$. The lower figures in each set are the stationary solutions to the path-averaged equation, the statistical analogs of no-noise solitary waves.

As the pinning period is decreased, the pulse width also decreases, as the pulse shape approaches that of an ideal soliton for model A. The pulse broadening is more intense for higher values of the noise strength (compare the left and right columns of pictures $D=0.1$ and $D=2.5$.)

One observes similar patterns in the case of dispersion management, with an additional feature. As noise is increased, the width of the central peak, as well as that for the sideband peaks, increases.

\section{DIRECT NUMERICAL SIMULATIONS}

We performed numerical investigations of both models $\mathrm{A}$ and $\mathrm{B}$ with $\xi$ and $\xi=\xi_{p}$ in the intermediate case, $z_{\mathrm{NL}} \sim z_{\xi}$. The initial $(z=0)$ pulse is an exact soliton for model A, and it is a Gaussian pulse close to the respective DM soliton for model B. (See also Refs. 28 and 30.) The Fourier split-step scheme with $2^{13}$ temporal Fourier modes and periodic boundary conditions is implemented on the domain $t \in[-180,180]$. The spatial step is $z_{\text {step }}=0.01$, and the numerical convergence is checked by varying the size of the temporal domain and number of the Fourier harmonics. Parameters for the initial signal were chosen to be $d_{0}=1, a=1$ in model $\mathrm{A}$, and $d_{0}$ $=0.15, \quad d_{\mathrm{DM}}=0.1, \quad z_{\mathrm{DM}}=1, \quad$ and $\quad|\psi(0 ; t)|$ $=0.79 \exp \left(-t^{2} / 2.6\right)$ in model B. The setup in model B is borrowed from Ref. 31 and corresponds to experimentally available DM fibers. Gaussian zero-mean noise correlated at $z_{\mathrm{var}}=0.1$ with amplitude $d_{\mathrm{var}}=1$ approximates the $\delta$-correlated uniform noise with $D=d_{\mathrm{var}}^{2} z_{\mathrm{var}}=0.1$.

We consider both natural noise, Eq. (3), and pinned noise, Eq. (4). Pinning strategies of two types are considered: strictly periodic, $l_{j+1}-l_{j}=l$, where $l$ is fixed, and quasi periodic, $l_{j+1}-l_{j}=l(1+\eta)$, where $\eta$ is a random
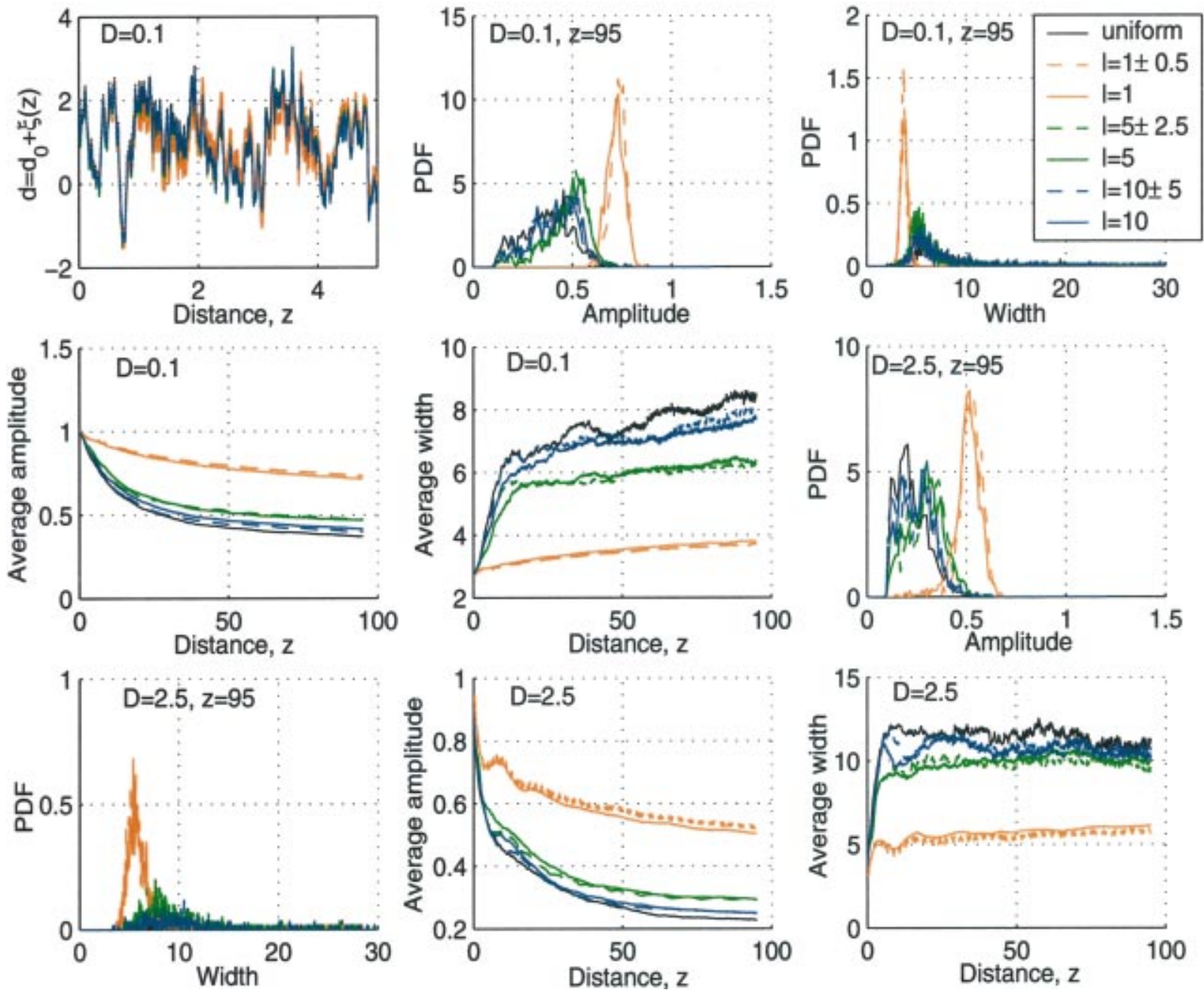

Fig. 3. Statistical description of the effect of pinning on optical pulse dynamics: constant-dispersion case (model A). The upper-left subfigure is a comparison of a pinned and unpinned random-dispersion profile. The upper center and right subfigures are PDFs for pulse amplitude and width, measured at distance $z=95$ for noise strength $D=0.1$, for various pinning lengths. The left and center subfigures in the middle row show the dependence of the average amplitude and width on the distance $z$ for various pinning lengths and noise strength $D=0.1$. The right subfigure of the middle row and the left subfigure of the bottom row represent PDFs of the pulse amplitude and width measured at distance $z=95$ for noise strength $D=2.5$ for various pinning lengths. The center and right subfigures of the bottom row show the dependence of the average amplitude and width on the distance $z$ for various pinning lengths and noise strength $D=2.5$. 

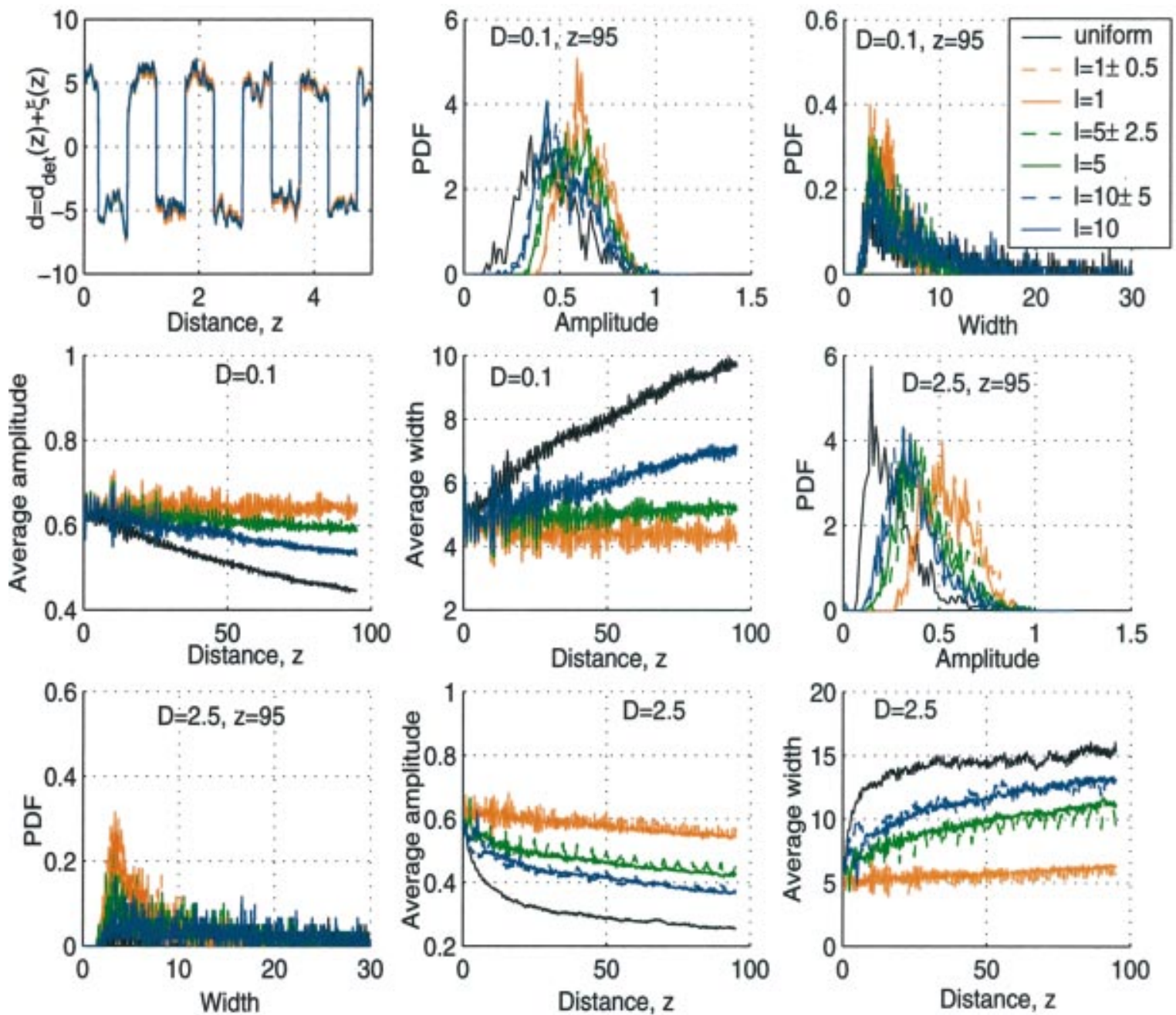

Fig. 4. Statistical description of the effect of pinning on the optical pulse dynamics: dispersion-management case (model B). The upper-left subfigure is a comparison of pinned and unpinned dispersion maps with randomness. The upper center and right subfigures are PDFs for pulse amplitude and width, measured at distance $z=95$ for noise strength $D=0.1$, for various pinning lengths. The left and center subfigures in the middle row show the dependence of the average amplitude and width on the distance $z$ for various pinning lengths and noise strength $D=0.1$. The right subfigure of the middle row and the left subfigure of the bottom row represent PDFs of the pulse amplitude and width measured at distance $z=95$ for noise strength $D=2.5$ for various pinning lengths. The center and right subfigures of the bottom row show the dependence of the average amplitude and width on the distance $z$ for various pinning lengths and noise strength $D=2.5$.

number uniformly distributed between $\pm 1 / 2$. The averaged (or otherwise strict) pinning period for the pinned case is taken to be 1,5 , or 10 . The simulations ran until $z=95$. Statistics were collected for $10^{4}$ realizations.

Each set of two figures (Figs. 1 and 3, and 2 and 4), for model A and model B, respectively, includes the following:

(1) the comparative plot of a single realization of dispersion for different types of pinning compensation;

(2) PDFs for pulse amplitude and pulse width (full width at half-maximum amplitude) at the end of the run $(z$ $=95)$;

(3) dependence of the average pulse amplitude and width on $z$, taken over 1000 realizations.

Solid black, red, green, and blue represent natural noise, and pinned noise with pinning periods $l=1,5,10$, respectively. The quasi-periodic curves are dashed and of the same color as the respective periodic ones. Numerics for two values of $D$ are shown: $D=0.1$ and $D$ $=2.5$.
For model A, all types of pinned noise demonstrate a significant reduction in the rate of pulse broadening compared with the natural case. The individual configurations that degrade (through pulse splitting, etc.) in the natural case maintain pulse integrity when each type of pinning compensation is applied. The dependence on the pinning period is monotonic: the peak amplitude of the pulse decays faster as the pinning period increases. The difference between the periodic and respective quasiperiodic cases is minor, with a slightly better confinement observed for the quasi-periodic case.

The destruction of the pulse is accompanied by the emission of continuous radiation by the soliton. The radiation is clearly seen in the movie made for individual runs (the movie is available at http:/cnls.lanl.gov/ chertkov/Fiber). Once the radiation reaches the boundaries of the numerical box, it reflects and starts to interfere with the still-localized solution (at $z \geq 20$ ). The latter (which is an effect of periodic, not vanishing, boundary 
conditions used in numerics) shows up in the change of the averaged-width behavior at larger distances, $z \sim 20$.

The effect of the pinned noise is more dramatic in the case of model B. For the case of pinning compensation with the averaged period $l=1$ (and also less) at $D$ $=0.1$, one observes a tendency toward statistically steady behavior: the average pulse width does not decay (in contrast to a decay in the uniform case), and the PDF of the pulse width and amplitude does not change shape with $z$. Notice, however, that for the case with the same pinning period $l=1$ but greater $D=2.5$, a minor, but still observable degradation of pulse occurred. This is consistent with the statement concerning the efficiency of the pinning made in Section 3: the greater $D$ is, the lower the critical pinning period. There is no visible emission of radiation by the localized solution for any case of model B. We have also checked that temporal and spatial averages (e.g., for the PDFs of the pulse width and amplitude) coincide in the steady case of $l=1, D=0.1$. Notice, however, that the size of the stationary PDFs support is wider than the respective average oscillations due to the periodic deterministic part of the dispersion (i.e., the process is not self-averaged, as it would be in the case of a weak noise, $D \ll 1$ ). The dependence on the type of compensation (for $l>1, D=0.1$, and all the cases considered for $D=2.5$ ) is monotonic, and the difference between the random and quasi-random cases is again minor. We conclude that the dramatic reduction in pulse decay due to pinning is the result of minor changes in the dispersion profile.

\section{CONCLUSIONS}

We demonstrated that randomness of chromatic dispersion, quantified experimentally in real fibers by Mollenauer and his coauthors, ${ }^{22,23}$ causes pulse degradation in optical fibers. This effect is proportional to the strength of disorder and inversely proportional to the fourth power of the pulse width. Therefore the noise in dispersion presents a potential source of serious limitation for the next generation of high-speed communications. This is a new example of the negative effect of fiber randomness on high-speed data transmission, in addition to the well known effect of polarization mode dispersion.

We proposed and developed the theoretical background of the pinning method, which prevents pulse deterioration and is capable of improving performance of high-speed optical fiber links. These theoretical findings were verified by direct numerical simulations. The pinning method consists of periodic compensation of accumulated fiber dispersion by insertion of an additional piece of fiber with a well controlled length and dispersion value. All components required for implementation of this method, including measurements of accumulated dispersion of a fiber span, are standard and well established in optical fiber communications. The pinning method can be implemented both for the upgrading of existing links and for the production of new optical cables. The pinning method is effective in optical fibers with and without dispersion management.

\section{ACKNOWLEDGMENTS}

We are grateful to G. D. Doolen, G. Falkovich, I. Fatkullin, J. Hesthaven, I. Kolokolov, V. Lebedev, P. Mamyshev, F. G. Omenetto, H. Rose, T. Schaefer, and S. Tretiak for constructive comments. The support of Laboratory Directed Research and Development Exploratory Research "Statistical Physics of Fiber Communications" at Los Alamos National Laboratory, a J. R. Oppenheimer fellowship (M. Chentkov), the U.S. Department of Energy through contract W-7-405-ENG-36 and its Program in Applied Mathematical Sciences, KJ-01-01 (I. Gabitov), U.S. Army Research Office, Far East (grant N62649-01-1-0002) (I. Gabitov), and National Science Foundation grant DMS0073923 (J. Moeser) is greatly acknowledged.

\section{APPENDIX A: DIFFUSION OF OPTICAL PULSE WIDTH AND EXISTENCE OF STATISTICAL STEADY STATE FOR NONLINEAR SCHRODINGER EQUATION WITH RANDOM DISPERSION}

In this appendix, we consider model $\mathrm{A}\left(d_{\operatorname{det}}=d_{0}\right)$ with natural noise (3). We prove that steady propagation of an optical pulse is not possible for nonpositive residual dispersion $d_{0}$. Moreover, we show that, for either arbitrary initial condition $\left.\psi\right|_{z=0}$ and $d_{0}=0$ or for an initial condition with no phase modulation and $d_{0} \leqslant 0$, the $z$ dependence of a statistically averaged root-mean-square pulse width $\left\langle T_{\mathrm{RMS}}^{2}\right\rangle \equiv\left\langle\int t^{2}|\psi|^{2} \mathrm{~d} t\right\rangle / P$ grows with $z$. (Here, the time-averaged optical power $P \equiv \int|\psi|^{2} \mathrm{~d} t$ is an integral of motion: $P_{z}=0$ ). We note that $\left\langle T_{\mathrm{RMS}}^{2}\right\rangle$ is a growing function of $z$ even for a small positive $d_{0}$; thus variations in dispersion result in diffusion of the optical pulse width.

Consider the following integrated (over $t$ ) object, $A$ $\equiv \int t^{2}|\psi|^{2} \mathrm{~d} t$. Integrating Eq. (1) by parts and applying vanishing boundary conditions at infinity, one derives

$$
A_{z}=\left(d_{0}+\xi\right) B, B \equiv \int 2 i t\left(\psi \psi_{t}^{*}-\psi^{*} \psi_{t}\right) \mathrm{d} t .
$$

In a similar way, differentiating $B$ with respect to $z$, one gets

$$
B_{z}=8\left[\left(d_{0}+\xi(z)\right)\right] X-4 Y,
$$

where $X \equiv \int\left|\psi_{t}\right|^{2} \mathrm{~d} t, Y \equiv \int|\psi|^{4} \mathrm{~d} t$. Note that Eq. (A2) coincides for $\xi(z)=$ constant with the so-called virial theorem for the $1+1$ NLS (see, e.g., Refs. 41-46). Assuming that the probability distribution function (PDF) for $\psi$ is $z$ independent, one derives $\left\langle B_{z}\right\rangle=0$. Then the statistical average of Eq. (A2) results in

$$
2 d_{0}\langle X\rangle+2\langle\xi X\rangle-\langle Y\rangle=0 .
$$

One checks that $\langle\xi X\rangle$ is zero for the short-correlated process (3). (A more accurate statement would be that the average vanishes with $z_{\mathrm{var}} \rightarrow 0$, where $z_{\text {var }}$ is correlation length of a physical, short, but finite correlated $\xi$.) Taking into account that $X$ and $Y$ are positive, one finds that a statistical steady state is forbidden for nonpositive average dispersion $d_{0} \leqslant 0$. Notice that the necessary condition for the steady state,

$$
2 d_{0}\langle X\rangle=\langle Y\rangle,
$$


is satisfied exactly for the one-soliton solution of the nonoise NLS, ${ }^{26,27}$ where the dispersion $d_{0}$ and the nonlinearity continuously balance each other. In this case the averages in Eq. (A4) are replaced by $X$ and $Y$, respectively. Therefore the lack of a statistically steady state for $d_{0}$ $\leqslant 0$ corresponds to the nonexistence of the soliton solution of the defocusing $\left(d_{0} \leqslant 0\right) \mathrm{NLS}^{26,27}$ Moreover, the relation (A4) tells us that in some averaged (integral) sense a one-pulse solution of Eq. (1), $\psi$, has to be close to a single-soliton solution of the no-noise NLS, if the onepulse solution is a statistically steady state. A quantummechanical interpretation of the NLS, where $d_{0} X / P$ is a kinetic energy and $-2 Y / P$ is a potential energy, suggests that Eq. (A4) can also be called an averaged virial theorem.

Equations (A1) and (A2) allow us to relate the derivative of $A_{z}$ to $X$ and $Y$ :

$$
\begin{aligned}
A_{z}=\left[\xi(z)+d_{0}\right] B(0)+8\left[\xi(z)+d_{0}\right] \int_{0}^{z}\left[\xi\left(z^{\prime}\right)\right. \\
\left.+d_{0}\right] X\left(z^{\prime}\right) \mathrm{d} z^{\prime}-4\left[\xi(z)+d_{0}\right] \int_{0}^{z} Y\left(z^{\prime}\right) \mathrm{d} z^{\prime} .
\end{aligned}
$$

Averaging Eq. (A5), one finds that various averages from the right-hand side of Eq. (A5), containing one multiplier $\xi$, vanish due to the casuality constraint. (This is valid only for the $\delta$ correlated limit, Eq. (3), of a physical process with the correlation scale, $z_{\mathrm{var}}$, going to zero.) The average of the only term on the right-hand side of Eq. (A5) containing the product of two (also taken at different $z$ ) $\xi$ is given by $4 D\langle X(z)\rangle$. One derives the following interesting constraint on the averaged pulse width squared:

$$
\begin{aligned}
\langle A\rangle_{z}= & d_{0}\langle B(0)\rangle+8 d_{0}^{2} \int_{0}^{z}\left\langle X\left(z^{\prime}\right)\right\rangle \mathrm{d} z^{\prime}+4 D\langle X(z)\rangle \\
& -4 d_{0} \int_{0}^{z}\left\langle Y\left(z^{\prime}\right)\right\rangle \mathrm{d} z^{\prime} .
\end{aligned}
$$

If $d_{0}=0$ (the case of dispersion-shifted fiber), $\langle A\rangle_{z}$ $=4 D\langle X(z)\rangle$, and, due to the positivity of $X$, one concludes that $\langle A\rangle$ grows with $z$. $X$ does not depend on $z$ in the linear case (see, also, Appendix B), and the growth of $\langle A\rangle$ with $z$ is linear (i.e., the pulse width experiences diffusive growth caused by random variations of dispersion coefficient). Nonlinearity is responsible for nontrivial dependence of $\langle X\rangle$ on $z$ and therefore for modification of the (still) diffusive law. Estimating $X, P$, and $D$ by $p^{2} / b, p^{2} b$, and $d_{\mathrm{var}}^{2} z_{\mathrm{var}}$ (where $d_{\mathrm{var}}$ and $z_{\mathrm{var}}$ stand for amplitude and length of the dispersion variations), respectively, one derives

$$
\beta \equiv\left(\left\langle T_{\mathrm{RMS}}(z)^{2}\right\rangle-T_{\mathrm{RMS}}(0)^{2}\right) / T_{\mathrm{RMS}}(0)^{2} \sim d_{\mathrm{var}}^{2} z_{\mathrm{var}} z / b^{4} .
$$

Then the minimal requirement for small information loss, $\beta \lesssim 1$, becomes $b \gtrsim 6 \mathrm{ps}$ for $z \sim 10^{4} \mathrm{~km}$ and the typical values of the dispersion variation length and amplitude, $z_{\mathrm{var}} \sim 100 \mathrm{~m}, d_{\mathrm{var}} \sim 1 \mathrm{ps}^{2} / \mathrm{km}$, corresponding to the aforementioned experiments. ${ }^{22,23}$ It suggests that construction of high-bit-error-rate lines based on the dispersion-shifted fiber requires essential improvement of fiber-production technology (and/or implementation of the pinning method).

\section{APPENDIX B: LINEAR PROBLEM}

This appendix is devoted to the exact analysis of the linear stochastic problem

$$
-i \partial_{z} \psi=d(z) \partial_{t}^{2} \psi
$$

A general solution of Eq. (B1) has the form

$$
\begin{aligned}
\psi(t ; z)= & \int_{-\infty}^{+\infty} \mathrm{d} k \int_{-\infty}^{+\infty} \frac{\mathrm{d} t^{\prime}}{2 \pi} \exp \left[i k\left(t-t^{\prime}\right)\right. \\
& \left.-i k^{2} \int_{0}^{z} \mathrm{~d}\left(z^{\prime}\right) \mathrm{d} z^{\prime}\right] \psi\left(t^{\prime} ; 0\right) .
\end{aligned}
$$

Considering the Gaussian initial pulse, $\psi(t ; 0)$ $=a_{0} \exp \left[-\left(t / b_{0}\right)^{2}+i \lambda_{0}+i t^{2} \mu_{0}\right]$, where $a_{0}, b_{0}, \lambda_{0}$, and $\mu_{0}$ are the initial amplitude, width, phase, and chirp of the pulse, one derives

$$
\begin{aligned}
\psi(t ; z)= & a_{0} \exp \left(i \lambda_{0}\right) \\
& \times \exp \left[-t^{2} \frac{1-i b_{0}^{2} \mu_{0}}{b_{0}^{2}+4\left(i+b_{0}^{2} \mu_{0}\right) \int_{0}^{z} \mathrm{~d}\left(z^{\prime}\right) \mathrm{d} z^{\prime}}\right] \\
& \times\left[1+4\left(i / b_{0}^{2}+\mu\right) \int_{0}^{z} \mathrm{~d}\left(z^{\prime}\right) \mathrm{d} z^{\prime}\right]^{-1} \cdot
\end{aligned}
$$

Equation (B3) shows that the Gaussian shape of the pulse does not change during propagation. Therefore the dynamics can be explained in terms of the following four parameters only:

$$
\begin{aligned}
& a(z)=a_{0} \operatorname{abs}\left\{\left[1+4\left(i / b_{0}^{2}+\mu\right) \int_{0}^{z} \mathrm{~d}\left(z^{\prime}\right) \mathrm{d} z^{\prime}\right]^{-1 / 2}\right\}, \\
& \lambda(z)=\lambda_{0}+\arg \left\{\left[1+4\left(i / b_{0}^{2}+\mu\right) \int_{0}^{z} \mathrm{~d}\left(z^{\prime}\right) \mathrm{d} z^{\prime}\right]^{-1 / 2}\right\},
\end{aligned}
$$

$b(z)$

$$
=\sqrt{b_{0}^{2}\left[1+4 \mu_{0} \int_{0}^{z} \mathrm{~d}\left(z^{\prime}\right) \mathrm{d} z^{\prime}\right]^{2}+\frac{16}{b_{0}^{2}}\left[\int_{0}^{z} \mathrm{~d}\left(z^{\prime}\right) \mathrm{d} z^{\prime}\right]^{2}}
$$

$$
\mu(z)=\frac{b_{0}^{4} \mu_{0}+4\left(1+b_{0}^{4} \mu_{0}^{2}\right) \int_{0}^{z} \mathrm{~d}\left(z^{\prime}\right) \mathrm{d} z^{\prime}}{b_{0}^{4}\left[1+4 \mu_{0} \int_{0}^{z} \mathrm{~d}\left(z^{\prime}\right) \mathrm{d} z^{\prime}\right]^{2}+16\left[\int_{0}^{z} \mathrm{~d}\left(z^{\prime}\right) \mathrm{d} z^{\prime}\right]^{2}} .
$$

Reduction from the field variable $\psi(t ; z)$ to the four functions of $z$ makes it easy to calculate the PDFs, which describe the transition probabilities from $z=0$ to $z=l$. We present here PDFs of the width, amplitude, and chirp calculated for the case of model A and natural noise (3), 


$$
\begin{aligned}
& \mathcal{P}\left(a_{l} \mid l ; a_{0}, b_{0}, \mu_{0}\right)=\sqrt{2} a_{0}^{4}\left(a_{l}^{10} \pi D l\left\{\mu_{0}^{2}+\left[\left(a_{0} / a_{l}\right)^{4}-1\right]\left(\mu_{0}^{2}+1 / b_{0}^{4}\right)\right\}\right)^{-1 / 2} \\
& \times \sum_{k=1 ; 2} \exp \left[-\frac{\left(\mu_{0}\left\{1+(-1)^{k} \sqrt{1+\left[\left(a_{0} / a_{l}\right)^{4}-1\right]\left[1+1 /\left(\mu_{0}^{2} b_{0}^{4}\right)\right]}\right\}\left[4\left(\mu_{0}^{2}+1 / b_{0}^{4}\right)\right]^{-1}+d_{0} l\right)^{2}}{2 D l}\right] . \\
& \mathcal{P}\left(b_{l} \mid l ; a_{0}, b_{0}, \mu_{0}\right)=b_{l}\left(32 \pi D l\left[\mu_{0}^{2} b_{0}^{4}+\left(b_{l}^{2} / b_{0}^{2}-1\right)\left(1+b_{0}^{4} \mu_{0}^{2}\right)\right]\right)^{-1 / 2} \\
& \times \sum_{k=1 ; 2} \exp \left\{-\frac{\left(\mu_{0} b_{0}^{4}\left[1+(-1)^{k} \sqrt{1+\left(b_{l}^{2} / b_{0}^{2}-1\right)\left(1+1 / b_{0}^{4} \mu_{0}^{2}\right)}\right]\left[4\left(1+b_{0}^{4} \mu_{0}^{2}\right)\right]^{-1}+d_{0} l\right)^{2}}{2 D l}\right\}, \\
& \mathcal{P}\left(\mu_{l} \mid l ; a_{0}, b_{0}, \mu_{0}\right)=\sum_{k=1 ; 2}\left[\frac{1+b_{0}^{4} \mu_{0}^{2}}{\sqrt{\left(1+b_{0}^{4} \mu_{0}^{2}\right)^{2}-4 b_{0}^{4} \mu_{l}^{2}}}+(-1)^{k}\right]\left(128 \mu_{l}^{4} \pi D l\right)^{-1} \\
& \times \exp \left\{-\frac{1}{2 D l}\left[\frac{1+b_{0}^{4} \mu_{0}\left(\mu_{0}-2 \mu_{l}\right)-(-1)^{k} \sqrt{\left(1+b_{0}^{4} \mu_{0}^{2}\right)^{2}-4 b_{0}^{4} \mu_{l}^{2}}}{8 \mu_{l}\left(1+b_{0}^{4} \mu_{0}^{2}\right)}-d_{0} l\right]^{2}\right\} .
\end{aligned}
$$

\section{APPENDIX C: ON THE DISTRIBUTION OF RETURNS TO THE ORIGIN FOR THE CONTINUOUS RANDOM WALK}

The question that we are going to answer is the following: What is the probability that the "integral" random walker, $h(z)=\int_{0}^{z} \mathrm{~d} z^{\prime} \xi\left(z^{\prime}\right)$, with $\xi(z)$ being the natural process described by Eq. (3), will return to the origin at position $Z$ for the first time, given it started from the origin at $z=0$ ? Similar accounts can be found in many places in the random-walk literature. See, for example, Ref. 47. ( $Z$ and $h$, here, play the role of time and displacement, respectively, in traditional random-walk theory.)

The probability distribution for the Brownian walker is a simple Gaussian:

$$
p(h, z)=\frac{1}{\sqrt{4 \pi D z}} \exp \left(-\frac{h^{2}}{4 D z}\right) .
$$

Our quantity to be determined is simply the first-passage distance (time, in the random-walk theory) distribution, $f(h, z)$, the probability that the walker visits point $h$ at distance $z$ for the first time, given it started from $h_{0}=0$ at $z_{0}=0$. We have, from the theory of recurrent events,

$$
p(h, z)=\int_{0}^{z} \mathrm{~d} z^{\prime} f\left(h, z^{\prime}\right) p\left(0, z-z^{\prime}\right) .
$$

Equation (C2) is solved by taking the Laplace transform, which for a function $g(z)$ is defined by

$$
\hat{g}(u)=\int_{0}^{\infty} \mathrm{d} z \exp (-u z) g(z), \quad \operatorname{Re}(u)>0 .
$$

Applying this to Eq. (C2), one obtains

$$
\hat{p}(x, u)=\hat{f}(x, u) \hat{p}(0, u) .
$$

The Laplace transform of Eq. (C1) is just

$$
\hat{p}(x, u)=\frac{1}{\sqrt{4 D u}} \exp \left(-\sqrt{\frac{u x^{2}}{D}}\right),
$$

and thus

$$
\hat{f}(x, u)=\exp \left(-\sqrt{\frac{u x^{2}}{D}}\right) .
$$

After performing the inverse transform, the result is the so-called Smirnov density:

$$
f(h, z)=\sqrt{\frac{h^{2}}{4 \pi D z^{3}}} \exp \left(-\frac{h^{2}}{4 D z}\right), \quad t>0 .
$$

This density is normalized, $\int_{0}^{\infty} \mathrm{d} z f(h, z)=1$. However, it exhibits an essential singularity at $z \rightarrow 0^{+}$. At large times, $z \rightarrow \infty$,

$$
f(h, z) \simeq \frac{|h|}{2 \sqrt{\pi D}} z^{-3 / 2} .
$$

If one looks for the first-passage distance distribution of returns, one obtains $f(0, z)=0$. This means that once the Brownian walker takes off from the origin, the number of paths that will return is vanishingly small compared with the total number of paths. This result does not allow us to count the returning paths to the origin within this normalization of the continuum process. These observations are also suggested by the fact that the average $z$ to reach point $h$ from the origin is $\infty$ :

$$
\begin{aligned}
\int_{0}^{z} \mathrm{~d} z z f(h, z)= & \frac{2}{\sqrt{\pi}} \sqrt{\frac{h^{2}}{4 D}} Z^{1 / 2} \exp \left(-\frac{h^{2}}{4 D Z}\right) \\
& -\frac{h^{2}}{2 D}\left\{1-\Phi\left[\left(\frac{h^{2}}{4 D}\right)^{1 / 4}\right]\right\},
\end{aligned}
$$

which diverges as $\sqrt{Z}$ [ $\Phi(x)$ is the error function]. The alternative solution is to analyze this question based on lattice walks or to introduce a proper regularization. On a lattice walk the first-passage distance probability is defined as the probability that the walker starting from the origin will reach site $s$, on the $n$th step, $F_{n}(s)$. We have $\sum_{j=0}^{n} F_{j}(s)=R_{n}(s)$ as the probability that site $s$ is reached 
(at all) during the first $n$ steps. The conditional mean first-passage distance for a walk of finite duration $n$ is the average distance to reach site $s$ for the first time during the first $n$ steps normalized to the walks that will eventually reach site $s$ during the first $n$ steps:

$$
\tau_{n}(s)=\sum_{j=0}^{n} \frac{j F_{j}(s)}{R_{n}(s)} .
$$

In one dimension the generating function for the firstpassage probabilities $F_{j}(s)$ is given by ${ }^{47}$

$$
F(s ; \xi)=\left\{\begin{array}{ll}
1-\sqrt{1-\xi^{2}}, & s=0 \\
\left(\frac{1-\sqrt{1-\xi^{2}}}{\xi}\right)^{|s|}, & s \neq 0
\end{array} .\right.
$$

The (unconditional) mean first-passage time is given by

$$
\tau_{\infty}(s)=\sum_{j=1}^{\infty} j F_{j}(s)=\left.\frac{\partial}{\partial \xi} F(s ; \xi)\right|_{\xi \rightarrow 1^{-}},
$$

which diverges just as in the case of the continuum Brownian walk. However, the conditional (conditional upon arriving) first-passage distance defined above will be given by

$$
\tau_{n}(s)=\left\{\begin{array}{ll}
\sqrt{\frac{2}{\pi}} \sqrt{n}, & s=0 \\
2 \pi|s| \sqrt{\frac{2}{\pi}} \sqrt{n}, & s \neq 0
\end{array} .\right.
$$

\section{REFERENCES}

1. A. Hasegawa and F. Tappert, "Transmission of stationary nonlinear optical pulses in dispersive dielectric fibers. I. Anomalous dispersion," Apl. Phys. Lett. 23, 142-144 (1973).

2. S. G. Evangelides, B. M. Nyman, G. T. Harvey, L. F. Mollenauer, P. V. Mamyshev, M. L. Saylors, S. K. Korotky, U. Koren, T. A. Strasser, J. J. Veselka, J. D. Evankow, A. Lucero, J. Nagel, J. Sulhoff, J. Zyskind, P. C. Corbett, M. A. Mills, and G. A. Ferguson, "Soliton WDM transmission with and without guiding filters," IEEE Photonics Technol. Lett. 8, 1409-1411 (1996).

3. L. F. Mollenauer, P. V. Mamyshev, and M. J. Neubelt, "Demonstration of soliton WDM transmission at 6 and 7 $\times 10 \mathrm{Gbit} / \mathrm{s}$, error free over transoceanic distances," Electron. Lett. 32, 471-473 (1996).

4. M. Nakazawa, K. Suzuki, H. Kubota, A. Sahara, and E. Yamada, "100Gbit/s WDM $(20 \mathrm{Gbit} / \mathrm{s} \times 5$ channels $)$ soliton transmission over $10000 \mathrm{~km}$ using in-line synchronous modulation and optical filtering," Electron. Lett. 33, 12331234 (1997)

5. C. Lin, H. Kogelnik, and L. G. Cohen, "Optical-pulse equalization of low-dispersion transmission in single-mode fibers in the 1.3-1.7- $\mu \mathrm{m}$ spectral region," Opt. Lett. 5, 476-478 (1980).

6. D. LeGuen, A. OHare, S. Del Burgo, D. Grot, F. Favre, and T. Georges, "Narrowband 640 Gbit/s soliton DWDM transmission over $1200 \mathrm{~km}$ of standard fibre with $100 \mathrm{~km}-21 \mathrm{~dB}$ amplifier spans," Electron. Lett. 34, 2345-2346 (1998).

7. D. LeGuen, S. Del Burgo, L. Moulinard, D. Grot, M. Henry, F. Favre, and T. Georges, "Narrow band 1.02 Tbit/s $\left(51^{*} 20 \mathrm{Gbit} / \mathrm{s}\right)$ soliton DWDM transmission over $1000 \mathrm{~km}$ of standard fiber with $100 \mathrm{~km}$ amplifier spans," in Optical Fiber Communication Conference, Postconference Digest, 1999 OSA Technical Digest Series (Optical Society of America, Washington, D.C., 1999), paper PD4-1.
8. L. F. Mollenauer, "Dispersion maps for ultra long distance, terabit capacity WDM," Conference on Lasers and ElectroOptics, Postconference Digest, Vol. 39 of OSA Trends in Optics and Photonics (Optical Society of America, Washington, D.C., 2000), p. 137.

9. L. F. Mollenauer, P. V. Mamyshev, J. Gripp, M. J. Neubelt, N. Mamysheva, L. Gruner-Nielsen, and T. Veng, "Demonstration of massive wavelength-division multiplexing over transoceanic distances by use of dispersion-managed solitons," Opt. Lett. 25, 704-706 (2000).

10. L. F. Mollenauer, K. Smith, J. P. Gordon, and C. R. Menyuk, "Resistance of solitons to the effects of polarization dispersion in optical fibers," Opt. Lett. 14, 1219-1221 (1989).

11. Xie Chongjin, M. Karlsson, H. Sennerud, and P. A. Andrekson, "Comparison of soliton robustness with respect to polarization-mode dispersion with first-order polarizationmode dispersion-compensated linear systems," Opt. Lett. 26, 672-674 (2001).

12. H. Sennerud, M. Karlsson, and P. A. Andrekson, "A comparison between NRZ and RZ data formats with respect to PMD-induced system degradation," IEEE Photonics Technol. Lett. 13, 448-450 (2001).

13. J. N. Elgin, "Inverse scattering theory with stochastic initial potentials," Phys. Lett. 110A, 441-443 (1985).

14. J. N. Elgin, "Perturbations of optical solitons," Phys. Rev. A 47, 4331-4341 (1993).

15. J. P. Gordon and H. A. Haus, "Random walk of coherently amplified solitons in optical fiber transmission," Opt. Lett. 11, 665-667 (1986).

16. G. Falkovich, I. Kolokolov, V. Lebedev, and S. Turitsyn, "Statistics of soliton-bearing systems with additive noise," Phys. Rev. E 63, 025601-1-025601-4 (2001).

17. C. D. Poole, "Statistical treatment of polarization dispersion in single-mode fiber," Opt. Lett. 13, 687-689 (1988).

18. C. D. Poole, J. H. Winters, and J. A. Nagel, "Dynamical equation for polarization dispersion," Opt. Lett. 16, 372374 (1991).

19. N. Gisin, "Solutions of the dynamical equation for polarization dispersion," Opt. Commun. 86, 371-373 (1991).

20. P. K. Wai, C. R. Menyuk, and H. H. Chen, "Stability of solitons in randomly varying birefringent fibers," Opt. Lett. 16, 1231-1233 (1991).

21. G. P. Agrawal, Fiber-Optic Communication Systems (Wiley, New York, 1997), Chap. 4, pp. 170-173.

22. L. F. Mollenauer, P. V. Mamyshev, M. J. Neubelt, "Method for facile and accurate measurement of optical fiber dispersion maps," Opt. Lett. 21, 1724-1726 (1996).

23. L. F. Mollenauer and J. Gripp, "Enhanced range for measurements of optical-time-domain-reflection-like dispersion map," Opt. Lett. 23, 1603-1605 (1998).

24. F. Omenetto, M. D. Moores, D. H. Reitza, and A. J. Taylor, "Adaptive control of femtosecond pulse propagation in optical fibers," Opt. Lett. 26, 938-940 (2001).

25. M. Chertkov, I. Gabitov, and J. Moeser, "Pulse confinement in optical fibers with random dispersion," Proc. Natl. Acad. Sci. U.S.A. 98, 14208-14211 (2001).

26. V. E. Zakharov and A. B. Shabat, "Exact theory of twodimensional self-focusing and one-dimensional selfmodulation of waves in nonlinear media," Zh. Eksp. Teor. Fiz. 61, 118-134 (1971).

27. V. E. Zakharov and A. B. Shabat, "Exact theory of twodimensional self-focusing and one-dimensional selfmodulation of waves in nonlinear media," Sov. Phys. JETP 34, 62-69 (1972).

28. I. Gabitov and S. K. Turitsyn, "Averaged pulse dynamics in a cascaded transmission system with passive dispersion compensation," Opt. Lett. 21, 327-329 (1996).

29. I. Gabitov and S. K. Turitsyn, "Breathing solitons in optical fiber links," JETP Lett. 63, 814-819 (1996).

30. N. Smith, F. M. Knox, N. J. Doran, K. J. Blow, and I. Bennion, "Enhanced power solitons in optical fiber transmission line," Electron. Lett. 32, 54-55 (1996).

31. S. K. Turitsyn, T. Schafer, K. H. Spatschek, and V. K. Mezentsev, "Path-averaged chirped optical soliton in dispersion-managed fiber communication lines," Opt. Commun. 163, 122-158 (1999). 
32. Y. Kodama, "Nonlinear chirped RZ and NRZ pulses in optical transmission lines," in Proceedings of the International Symposium on New Trends in Optical Soliton Transmission Systems, A. Hasegawa, ed. (Kluwer Academic, Boston, Mass., 1998), pp. 131-153.

33. N. J. Doran, W. Forysiak, and J. H. B. Nijhof, "Remarkable features of DM solitons: implications for high speed and WDM systems," in Proceedings of the International Symposium on New Trends in Optical Soliton Transmission Systems, A. Hasegawa, ed. (Kluwer Academic, Boston, Mass., 1998), pp. 303-316.

34. W. Feller, An Introduction to Probability Theory and Its Applications (Wiley, New York, 1957).

35. F. Kh. Abdullaev, J. G. Caputo, and M. P. Srensen, "Propagation and interaction of optical solitons in fibers with random parameters," in Proceedings of the International Symposium on New Trends in Optical Soliton Transmission Systems, A. Hasegawa, ed. (Kluwer Academic, Boston, Mass., 1998), pp. 1-13.

36. F. Kh. Abdullaev, J. C. Bronski, and G. Papanicolaou, "Soliton perturbations and the random Kepler problem," Physica D 135, 369-386 (2000)

37. F. Kh. Abdullaev and B. B. Baizakov, "Disintegration of a soliton in a dispersion-managed optical communication line with random parameters," Opt. Lett. 25, 93-95 (2000).
38. M. Chertkov, I. Gabitov, I. Kolokolov, and V. Lebedev, "Shedding and interaction of solitons in imperfect medium," JETP Lett. 74, 391-395 (2001).

39. R. Ohhira, A. Hasegawa, and Y. Kodama, "Methods of constructing a long-haul soliton transmission system with fibers having a distribution in dispersion," Opt. Lett. 20 701-703 (1995)

40. P. M. Lushnikov, "Dispersion-managed soliton in a strong dispersion map limit," Opt. Lett. 26, 1535-1537 (2001).

41. V. E. Zakharov, "Collapse of Langmuir waves," Zh. Eksp. Teor. Fiz. 62, 1745-1759 (1972).

42. V. E. Zakharov, "Collapse of Langmuir waves," Sov. Phys. JETP 35, 908-914 (1972).

43. P. M. Lushnikov, "Dynamic criterion for collapse," Pis'ma Zh. Eksp. Teor. Fiz. 62, 447-452 (1995).

44. P. M. Lushnikov, "Dynamic criterion for collapse," JETP Lett. 62, 461-467 (1995)

45. P. M. Lushnikov, "On the boundary of the dispersionmanaged soliton existence," Pis'ma Zh. Eksp. Teor. Fiz. 72 $163-167$ (2000).

46. P. M. Lushnikov, "On the boundary of the dispersionmanaged soliton existence," JETP Lett. 72, 111-114 (2000)

47. B. D. Hughes, Random Walks and Random Environments. Vol. 1. Random Walks (Clarendon, Oxford, 1995). 